\title{
Desinfectantes en aerosol como alternativa de control ambiental de los hongos anemófilos en viviendas
}

\author{
(Disinfectant spray as an alternative environmental control of airborne fungi in houses)
}

\author{
Aldo Tomás Costa ${ }^{1}$, Dante Javier Bueno ${ }^{2 *}$, Ricardo Azario ${ }^{1}$ \\ 1Facultad de Ciencias de la Salud, Universidad Nacional de Entre Ríos, \\ Lorenzo Sartorio 2160, 3260, Concepción del Uruguay, Entre Ríos, Argentina. \\ 2Instituto Nacional de Tecnología Agropecuaria (INTA), \\ Estación Experimental Agropecuaria Concepción del Uruguay, \\ Casilla de Correo № 6, 3260, Entre Ríos, Argentina. \\ *Autor para correspondencia: Tel/Fax: +54-3442-438073/74/75. C. \\ dantejb@yahoo.com.ar \\ RECIBIDO:25 de Noviembre de 2014 \\ APROBADO:13 de Marzo de 2015 \\ LOS AUTORES DECLARAN NO TENER CONFLICTO DE INTERESES
}

Palabras claves: desinfectantes en aerosol, hongos ambientales, casas.

Keywords: disinfectant spray, airborne fungi, houses.

\section{RESUMEN}

El objetivo de este trabajo fue evaluar el efecto de productos desinfectantes comerciales en aerosol sobre la carga de hongos ambientales en habitaciones de viviendas, teniendo en cuenta que las personas, principalmente los niños, pasan la mayor parte del día en las mismas. Además, se estudió, a través de encuestas, las preferencias del público a la hora de adquirir los desinfectantes comerciales ensayados. El número de UFC/placa fue variable dentro de la misma habitación para las distintas mediciones realizadas antes de la aplicación de los productos desinfectantes. Los porcentajes de inhibición del crecimiento fúngico fueron variables dependiendo del producto desinfectante ensayado y de la habitación muestreada. Dichos porcentajes no fueron diferentes significativamente entre los desinfectantes. Los valores de temperatura estuvieron dentro de un rango de $11 \mathrm{a}$ $19,5^{\circ} \mathrm{C}$ y el porcentaje de humedad relativa varió entre un 64 a $85 \%$. El $89 \%$ de los encuestados conocía la existencia de desinfectantes ambientales en aerosol. El 78\% conocía el Lysoform ${ }^{\circledR}$, seguido por Glade ${ }^{\circledR}$ antitabaco y Ayudin ${ }^{\circledR}$. Dentro de los productos más utilizados, el $54 \%$ de los encuestados indicó el producto Lysoform ${ }^{\circledR}$. La mayoría de los consumidores eligieron a los desinfectantes por lo que conocen y los medios de comunicación influyeron en el 29\% de los consumidores al momento de elegir el desinfectante. Estos productos ensayados son recomendables en una habitación para disminuir la carga de hongos ambientales y así reducir el efecto perjudicial de estos hongos sobre las personas que la utilizan.

\section{ABSTRACT}

The aim of this study was to evaluate the effect of commercial disinfectant spray on the concentration of airborne fungi in bedrooms, considering that people, especially children, spend most of the day in them. Also, public preferences through surveys were studied when acquiring commercial disinfectants 
Desinfectantes en aerosol como alternativa de control ambiental - A. Costa et al

tested. The number of CFU/Petri dish was variable within the same bedroom for the different measurements performed before application of the disinfectant. Percent inhibition of fungal growth were variable, depending on the disinfecting product tested and sampled bedroom. These percentages were not significantly different between the disinfectants. The temperature values were within a range of 11 to 19.5 ${ }^{\circ} \mathrm{C}$ and relative humidity percentage ranged from 64 to $85 \%$. Eighty nine percent of respondents knew about environmental disinfectant spray. Seventy-eight percent knew about Lysoform ${ }^{\circledR}$, followed by antismoking Glade $\AA$ and Ayudin $\AA$. Among the most widely used products, $54 \%$ of respondents said the Lysoform $\AA$ product was the most used. Most consumers chose known disinfectants and, when choosing a disinfectant, the media influenced $29 \%$ of consumers. The used products are recommended to be used in a bedroom to decrease the number of airborne fungi and thus reduce the harmful effect of these fungi on the people that use this room.

\section{INTRODUCCIÓN}

En el aire existe material particulado, el cual se puede originar de diversas fuentes, ya sea naturales 0 de procesos realizados por el hombre. Entre las fuentes naturales se destacan: la erosión de los suelos, las erupciones volcánicas, los incendios forestales, y algunas de tipo biológico como los granos de polen y microorganismos tales como bacterias, virus y esporas de hongos. Estos últimos, de dimensiones relativamente grandes, constituyen el grupo de hongos anemófilos, que al ser inhalados pueden provocar alergias y otras enfermedades en los individuos susceptibles (Strass et al., 2002; Barnes et al., 2006). Las poblaciones más susceptibles son los niños y ancianos, en menor grado, los adolescentes y adultos jóvenes (Lozano et al., 2003; Senent Sánchez, 2012).

Los hongos anemófilos se encuentran ampliamente distribuidos en la naturaleza y su ciclo de vida se desarrolla sobre materia orgánica muerta, ya que su función es la de «degradadores de materia orgánica». Sin embargo, la mayoría de los hongos presentes en los ambientes internos, como una vivienda, son saprofíticos, porque ellos obtienen lo que necesitan para su metabolismo de materiales muertos, materia orgánica o sustratos como madera, papel, pintura, polvo, piel y alimentos (Albright, 2001). Durante su ciclo biológico estos hongos producen esporas que le permiten dispersarse y reproducirse en condiciones ambientales que le son adversas. Las esporas, cuando encuentran condiciones de temperatura y humedad óptimas, se desarrollan produciendo miles de nuevas esporas que utilizan la materia orgánica presente en el ambiente dentro y fuera del domicilio (Yang y Johanning, 1997), constituyendo un riesgo para la salud.

Las condiciones ambientales de humedad relativa entre el 50 y $80 \%$, temperatura de 5 a $32^{\circ} \mathrm{C}$, precipitaciones, vientos, contaminación y actividades humanas influyen directamente en la propagación y proliferación de las partículas fúngicas hacia el interior de las viviendas. Por lo tanto, la importancia de estas partículas como agentes alergenos, radica no sólo en su capacidad de estar en los diferentes ambientes que rodean al ser humano, sino por su capacidad de invadir las mucosas respiratorias de huéspedes susceptibles (Burge et al., 2000; Horner et al., 1995; Portnoy et al., 2005; Sneller y Roby, 1979).

Al no poder eliminar completamente a estos hongos de los ambientes cerrados, existe una gran preocupación por el control de los mismos, para no llegar a límites críticos que causen problemas en la salud. Distintos productos desinfectantes se utilizan para controlar el crecimiento de los hongos. Estos productos pueden ser a base de alcohol, peróxido de hidrógeno, hipoclorito de sodio, amonio cuaternario, $\mathrm{y} / 0$ fenol (Madigan et al. , 2004). Existen en el mercado diversos productos desinfectantes en aerosol de venta libre y de bajo costo, que se dicen ser eficientes para el control de hongos ambientales. Sin embargo, se disponen de pocos datos acerca de su eficiencia. Por ello, en este trabajo se estudió la eficiencia de los productos desinfectantes comerciales en aerosol para disminuir la carga de hongos anemófilos en la habitación de viviendas ubicadas en la ciudad de Concepción del Uruguay, Entre Ríos, Argentina, teniendo en cuenta que las personas, principalmente los niños, pasan la mayor parte del día en las mismas. También, se evaluó las preferencias del público a la hora de adquirir los desinfectantes comerciales ensayados. 


\section{MATERIALES Y MÉTODOS}

\section{Productos desinfectantes}

Se utilizaron cuatro desinfectantes en aerosol de venta libre, con distintos principios activos (Figura 1): A) Glade ${ }^{\circledR}$ antitabaco; B) Lysoform ${ }^{\circledR}$; C) Ayudin $\AA$; y D) Procenex ${ }^{\circledR}$. Los mismos fueron adquiridos en supermercados de la ciudad de Concepción del Uruguay, Entre Ríos.

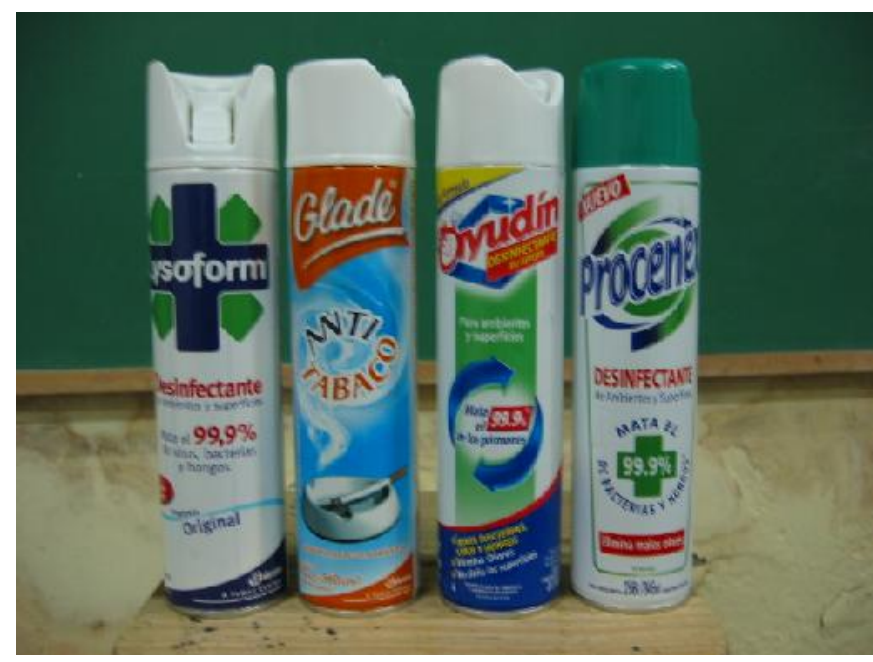

Figura 1.Desinfectantes comerciales en aerosol utilizados en el ensayo para estudiar el control de la carga de hongos ambientales.

\section{Viviendas y ambientes seleccionados para el ensayo de aplicación del desinfectante}

Se seleccionaron tres viviendas, ubicadas en diferentes zonas de Concepción del Uruguay, Entre Ríos (Figura 2). Las mismas tenían las siguientes características:

- $\quad$ Casa No 1: ubicada en zona portuaria.

- $\quad$ Casa No 2: ubicada en zona céntrica.

- $\quad$ Casa $N^{0} 3$ : lindera a una industria situada en una zona residencial.

Los factores de riesgo identificados para cada vivienda que facilitan la dispersión de los hongos anemófilos fueron:

- Casa 1: emisión de material particulado debido al tránsito de camiones, localización de molino harinero y almacenaje de granos. Presentaría mayor humedad relativa por su cercanía al río.
- Casa 2: emisiones gaseosas por el alto tránsito de vehículos en horario comercial.

- Casa 3: emisión de material particulado debido a una industria arrocera. Se registra un alto tránsito vehicular pesado y las calles linderas carecen de pavimento.

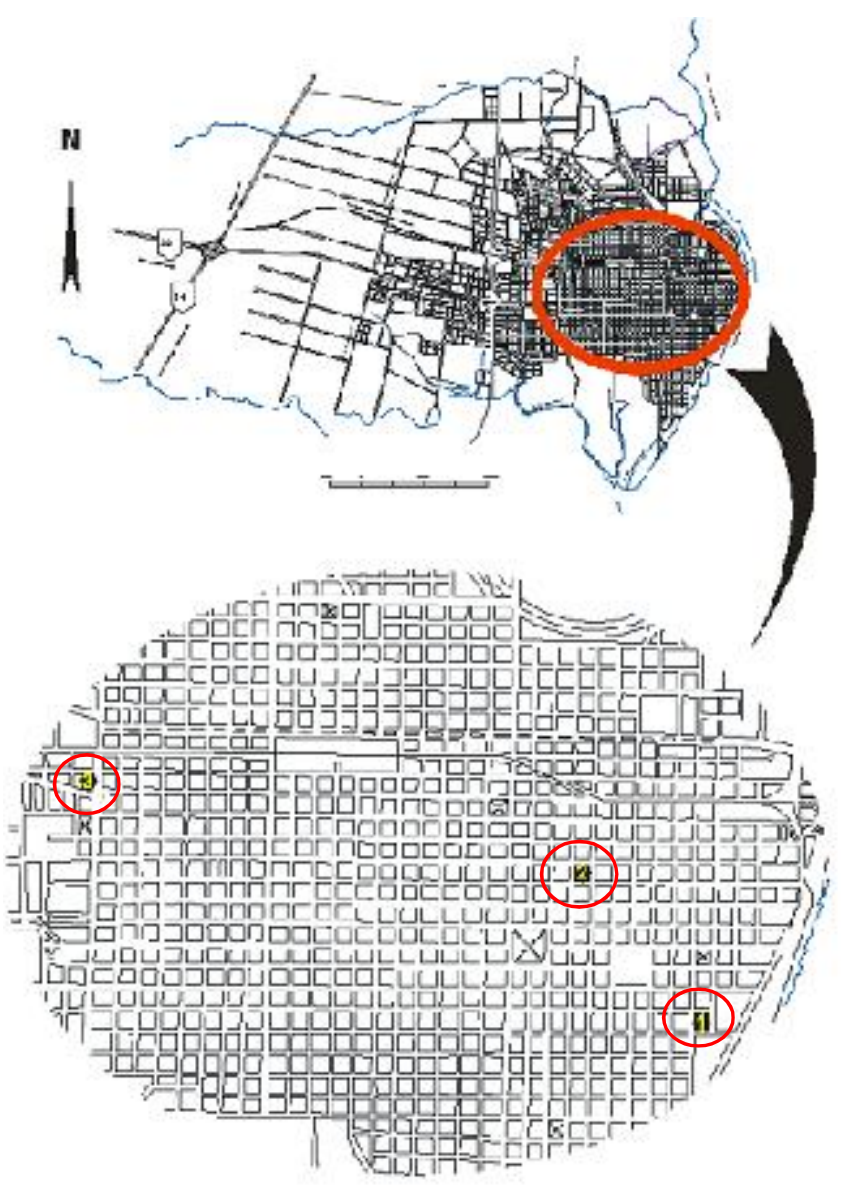

Figura 2. Ubicación de las 3 viviendas en Concepción del Uruguay, Entre Ríos, Argentina.(1) - Casa 1. (2) Casa 2. (3 - Casa 3.

Para realizar el ensayo de los desinfectantes ambientales se seleccionó un dormitorio, considerando que las personas duermen entre 6 y 8 horas, y así están más expuestas a los hongos ambientales. Las casas y habitaciones a ensayar tuvieron distintas características respecto a construcción, superficie, mantenimiento, calefacción y orientación de la ventana. En la Tabla 1 se detallan los materiales de construcción como factores que podrían influir en las condiciones ambientales necesarias para el desarrollo de la flora fúngica. 
Desinfectantes en aerosol como alternativa de control ambiental - A. Costa et al

Tabla 1. Características de construcción y limpieza de las 3 habitaciones (1 por cada casa) para la prueba con los desinfectantes ensayados.

\begin{tabular}{|l|l|l|l|}
\hline $\begin{array}{c}\text { Características de } \\
\text { construcción y mantenimiento }\end{array}$ & Habitación 1 & Habitación 2 & Habitación 3 \\
\hline Dimensiones $\left(\mathrm{m}^{3}\right)$ & 31,2 & 41,6 & 39 \\
Superficie ventanal $\left(\mathrm{m}^{2}\right)$ & 1,80 & 1,95 & 0,96 \\
Orientación de la ventana & Este & $\mathrm{Sur}$ & 0 este \\
Paredes revocadas & $\mathrm{Si}$ & $\mathrm{Si}$ & $\mathrm{Si}$ \\
Pisos & Cerámico & Cerámico & Cemento \\
Pintura de paredes & Látex & Látex & Cal \\
Calefacción & No & $\mathrm{Si}$ & No \\
Cielorraso & Madera & Yeso & Loza \\
Frecuencia de limpieza/ semana & 2 & 3 & 1 \\
\hline
\end{tabular}

\section{Ensayo sobre viviendas}

El ensayo se realizó en los meses de mayo y junio del 2007. Se siguió una técnica no volumétrica (gravimétrica) para el muestreo del aire, exposición por 10 minutos a las placas de Petri abiertas, conteniendo agar papa glucosada (Merck, Darmstadt, Alemania) con cloranfenicol $(0,25 \mathrm{~g} / \mathrm{l}$, Anedra, San Fernando, Argentina) como antibiótico, para inhibir la carga bacteriana ambiental. Las 3 placas fueron colocadas sobre la cama de una habitación (Figura 3). Posteriormente de cerrarse las placas de Petri, se aplicó uno de los desinfectantes ambientales seleccionados, teniendo en cuenta las especificaciones del envase (Figura 4). Luego de 10 minutos de finalizada la aplicación, se colocó 3 placas de Petri abiertas con el mismo medio de cultivo sobre las placas preexistentes y se las abrió por diez minutos (Figura 5). Todas estas placas fueron incubadas en estufa a $25-28{ }^{\circ} \mathrm{C}$ durante 7 días y cada colonia fúngica fue enumerada. Cada prueba se realizó una vez por producto desinfectante en cada habitación, midiendo la humedad relativa y temperatura de cada ambiente ensayado con un termómetro-higrómetro (Alla France, Chemillé, Francia) en cada muestreo. Se realizó un ensayo por semana para que se regenere la flora original que se inhibiría por la acción del desinfectante.

\section{Encuestas sobre el uso de los desinfectantes ambientales}

Se realizaron encuestas para identificar el producto más utilizado y la influencia de los medios de comunicación masivos sobre el consumo de los mismos. Las preguntas se detallan en la Tabla 2. Se encuestaron personas de ambos sexos entre 25 a 59 años de edad. El número de encuestas se obtuvo al aplicar la siguiente fórmula (Duffau, 1999):

$$
n=\frac{N * \mathrm{Z}_{\alpha}^{2} * p^{*} \mathrm{q}}{d^{2} *(N-1)+Z_{\alpha}^{2} * p^{*} q}
$$


Desinfectantes en aerosol como alternativa de control ambiental - A. Costa et al

$\mathbf{n}=$ tamaño de muestra.

$\mathbf{N}$ : tamaño de la población. Según estimaciones del Instituto Nacional de Estimaciones y Censo, en el 2007 Concepción del Uruguay tenía una población aproximada de 70.413 , del cual el 50,16\% (35.316 habitantes) tenía entre 25 y 59 años (Auditoría Ciudadana, 2006; INDEC, 2008).

$\mathbf{Z}_{\mathbf{a}}=$ coeficiente del nivel de confianza. Se tomó el valor de 1,645 correspondiente al nivel de confianza del $90 \%$. p: probabilidad de éxito o proporción esperada. Por no tener la información se tomó el valor de 0,5.

$\mathbf{q}=$ probabilidad de fracaso $=1-p$.

$\mathbf{d}=$ precisión. Se tomó el valor del 12\%.

El número de encuestas a realizar fue de 46 . Para ello, se seleccionaron aleatoriamente 15 personas en tres supermercados cercanos a las viviendas seleccionadas de la ciudad de Concepción del Uruguay. En un supermercado se encuestaron 16 personas.

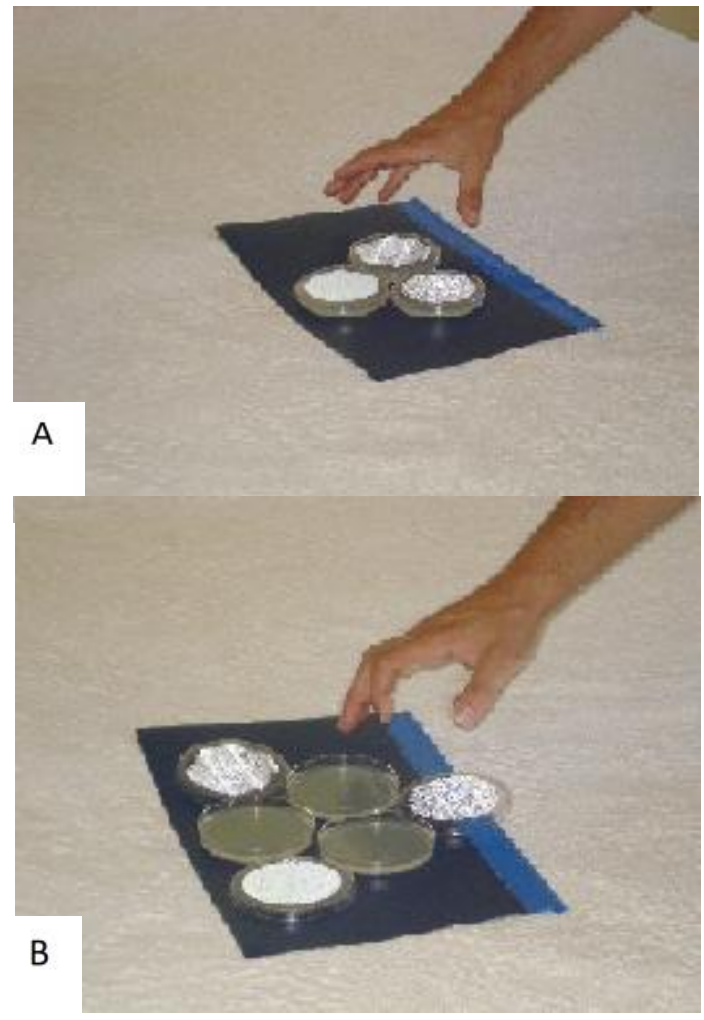

Figura 3. Disposición de las placas de Petri con agar papa glucosada con cloranfenicol antes de aplicar el producto desinfectante ambiental. A) Placas de Petri cerradas. B) Placas de Petri abiertas y expuestas por 10 minutos.

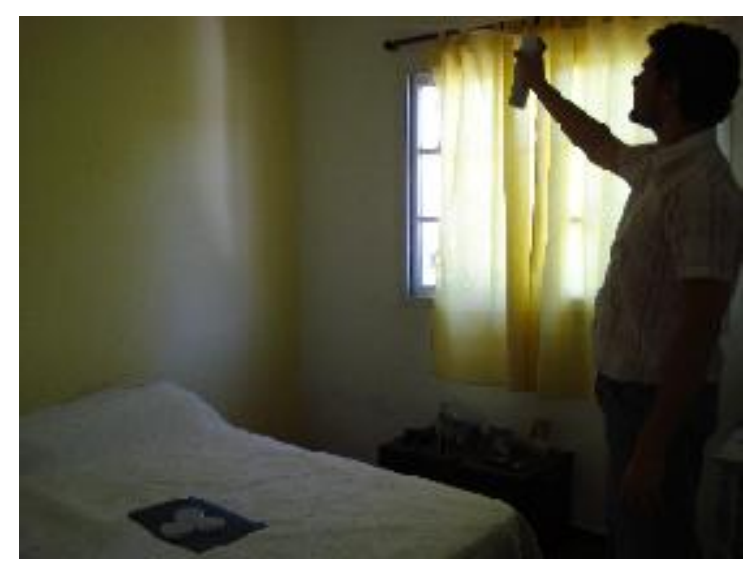

Figura 4. Aplicación del desinfectante según las indicaciones del embase.

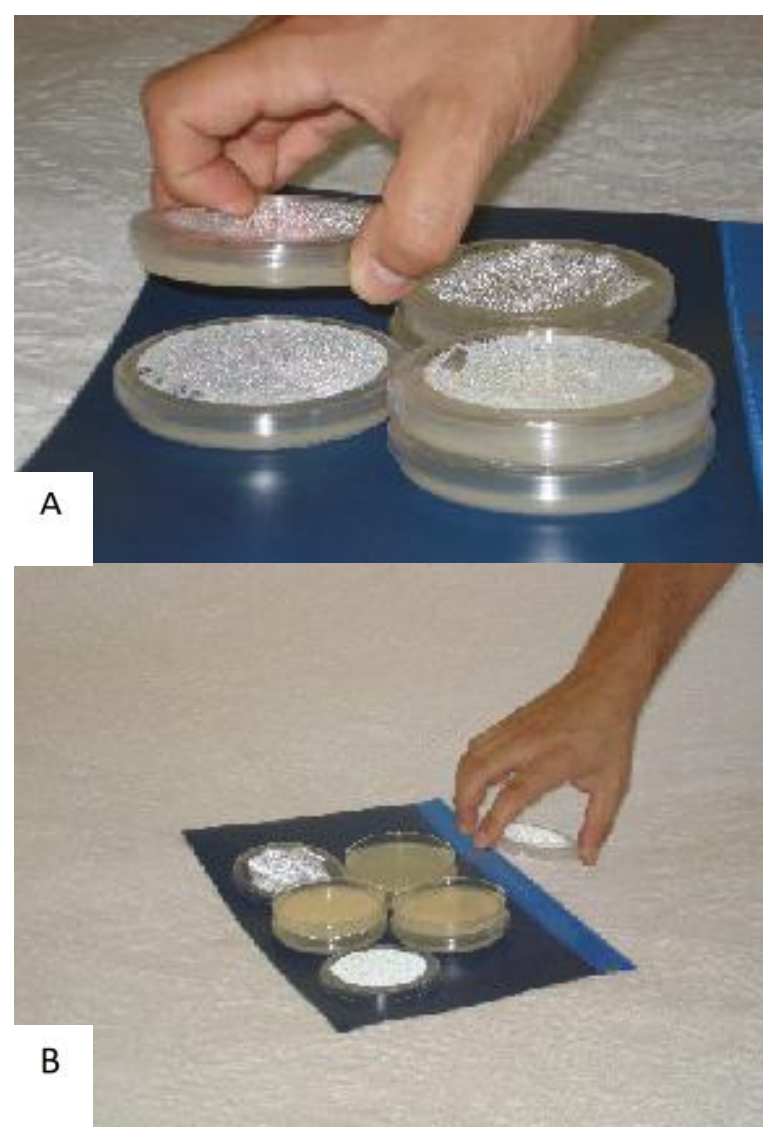

Figura 5. Disposición de las placas de Petri con agar papa glucosada con cloranfenicol después de aplicar el producto desinfectante ambiental A) Se colocaron las placas de Petri 10 minutos después de la aplicación del desinfectante sobre las otras para tomar la muestra en el mismo punto que el control. B) Se expusieron por 10 minutos las placas abiertas. 


\section{Métodos de análisis de datos}

La efectividad de los desinfectantes en la disminución de la carga fúngica ambiental fue determinada por el recuento en placa de las colonias formadas por los hongos anemófilos (UFC/placa) antes y después de la aplicación del desinfectante para cada caso. La media del recuento de las 3 placas de Petri fue utilizada para el análisis, según el siguiente cálculo:

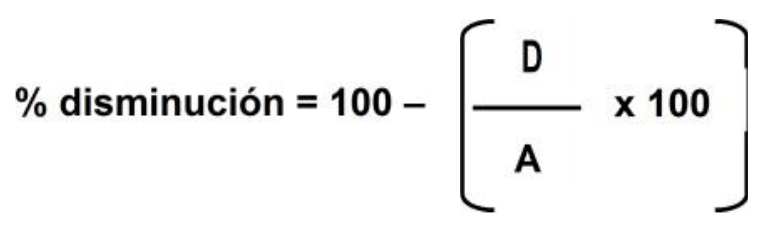

D: media de las UFC después del tratamiento con el desinfectante.

A: media de las UFC antes del tratamiento con el desinfectante

La comparación de la cantidad de UFC/placa antes y después de la aplicación del desinfectante en aerosol para cada casa y el porcentaje de disminución del número de UFC/placa de Petri entre los desinfectantes fue analizado con el test ANOVA de una vía. Las medias que mostraron diferencias significativas fueron comparadas usando el test de Tukey (Minitab Student R12). Todos los valores de significancia estadística están basados en un nivel de probabilidad de 0,05 (Rossman y Chance, 1998).

Tabla 2. Modelo de encuesta utilizado para conocer las preferencias del público.

\begin{tabular}{|l|l|l|}
\hline Preguntas & Posibles respuestas & Interpretación \\
\hline $\begin{array}{l}\text { ¿Conoce algún } \\
\text { desinfectante ambiental? }\end{array}$ & Si - No. & $\begin{array}{l}\text { Criterio de inclusión de las personas en } \\
\text { el estudio de desinfectantes ambientales } \\
\text { comerciales. Respuesta por «Sl» }\end{array}$ \\
\hline ¿Cuál? & $\begin{array}{l}\text {-Glade® antitabaco } \\
\text {-Lysoform }{ }^{\circledR} \\
\text {-Ayudin® } \\
\text { Procenex® }\end{array}$ & $\begin{array}{l}\text { Identificar cuál de los 4 principios activos } \\
\text { ensayado en el estudio es conocido por } \\
\text { la población. }\end{array}$ \\
\hline ¿Cuál utiliza? & $\begin{array}{l}\text {-Glade® antitabaco } \\
\text {-Lysoform }{ }^{\circledR} \\
\text {-Ayudin } \\
\text { Procenex® }\end{array}$ & $\begin{array}{l}\text { Conocer cuál de los 4 desinfectantes } \\
\text { estudiado es el más utilizado. }\end{array}$ \\
\hline ¿Por qué lo elige? & $\begin{array}{l}\text {-Es el más económico. } \\
\text {-Se lo recomendaron. } \\
\text {-Es el que conoce } \\
\text {-Por los buenos resultados que } \\
\text { se explicitan en los medios de } \\
\text { comunicación }\end{array}$ & $\begin{array}{l}\text { Conocer si los medios de comunicación } \\
\text { marcan la tendencia al momento de } \\
\text { elegir el/ los producto/s estudiados. }\end{array}$ \\
\hline
\end{tabular}


Desinfectantes en aerosol como alternativa de control ambiental - A. Costa et al

\section{RESULTADOS Y DISCUSIÓN}

El número de UFC/placa fue variable dentro de la misma habitación para las distintas mediciones realizadas antes de la aplicación de los productos desinfectantes (Tabla 3). Por otro lado, considerándose el número de UFC/placa se observó, en algunos casos, diferencia estadística entre el recuento de hongos antes y después de la aplicación del producto. La técnica de muestreo utilizada por gravedad 0 deposición es considerada un método no cuantitativo. La recolección de microorganismos ambientales por este método está afectada por el tamaño y la forma de las partículas y por el movimiento del aire circundante (Buttner y col., 1996). Sin embargo, en otros ambientes como en las plantas de incubación de huevos de aves, el muestreo gravimétrico permite clasificar el grado de limpieza de los lugares en base al número de microorganismos crecidos por Placa de Petri (Ernest, 1987).
A fin de disminuir la variación entre las muestras y poder hacer una comparación para cada ensayo, las placas fueron colocadas en la misma posición. Así también, se produjo escaso movimiento del aire entre el antes y el después de la aplicación del producto desinfectante. Sin embargo, las muestras con los distintos desinfectantes fueron tomadas en distintos días, y tanto las condiciones meteorológicas, el número de personas que frecuentaron la habitación y actividades de construcción en la parte de afuera de la habitación pudieron afectar la carga de hongos en ese lugar (Tormo-Molina y col., 2012).

Los porcentajes de inhibición del crecimiento fúngico fueron variables dependiendo del producto desinfectante ensayado y de la habitación muestreada (Tabla 3). Dichos porcentajes no fueron diferentes significativamente entre los desinfectantes $(P=0,75)$, promediando $44 \%, 55 \%, 61 \%$ y $64 \%$ de disminución del recuento de hongos ambientales para Glade ${ }^{\circledR}$ antitabaco, Lysoform ${ }^{\circledR}$, Procenex ${ }^{\circledR}$, y Ayudin ${ }^{\circledR}$,

Tabla 3. Número de hongos (UFC/placa) antes y después de la aplicación del desinfectante y \% de inhibición del número de hongos por estos productos.

\begin{tabular}{|c|c|c|c|c|}
\hline \multirow[t]{2}{*}{ Producto } & \multirow[t]{2}{*}{ Momento de medición } & \multicolumn{3}{|c|}{$\begin{array}{c}\text { Promedio } \pm \text { desvío estándar de las UFC/placa según } \\
\text { las habitaciones de las casa antes y después de la } \\
\text { aplicación de los desinfectantes comerciales en } \\
\text { aerosol (\% inhibición) }\end{array}$} \\
\hline & & Casa 1 & Casa 2 & Casa 3 \\
\hline Glade $®$ antitabaco & $\begin{array}{l}\text { Antes } \\
\text { Después }\end{array}$ & $\begin{array}{l}3 \pm 2^{\mathrm{a}} \\
1 \pm 2^{\mathrm{a}}(66,67)\end{array}$ & $\begin{array}{l}4 \pm 0^{a} \\
2 \pm 2^{a}(50,00)\end{array}$ & $\begin{array}{l}19 \pm 4^{a} \\
16 \pm 4^{a}(15,79)\end{array}$ \\
\hline Lysoform ${ }^{\circledR}$ & $\begin{array}{l}\text { Antes } \\
\text { Después }\end{array}$ & $\begin{array}{l}8 \pm 2^{\mathrm{a}} \\
2 \pm 1^{\mathrm{b}}(75,00)\end{array}$ & $\begin{array}{l}5 \pm 1^{\mathrm{a}} \\
2 \pm 1^{\mathrm{b}}(60,00)\end{array}$ & $\begin{array}{l}7 \pm 3^{a} \\
5 \pm 2^{a}(28,57)\end{array}$ \\
\hline Ayudin $\circledast$ & $\begin{array}{l}\text { Antes } \\
\text { Después }\end{array}$ & $\begin{array}{l}6 \pm 1^{\mathrm{a}} \\
3 \pm 2^{\mathrm{b}}(50,00)\end{array}$ & $\begin{array}{l}4 \pm 2^{\mathrm{a}} \\
1 \pm 1^{\mathrm{b}}(75,00)\end{array}$ & $\begin{array}{l}6 \pm 3^{a} \\
2 \pm 2^{a}(66,67)\end{array}$ \\
\hline Procenex® & $\begin{array}{l}\text { Antes } \\
\text { Después }\end{array}$ & $\begin{array}{l}21 \pm 15^{\mathrm{a}} \\
6 \pm 3^{\mathrm{a}}(71,43)\end{array}$ & $\begin{array}{l}8 \pm 6^{\mathrm{a}} \\
3 \pm 2^{\mathrm{a}}(62,50)\end{array}$ & $\begin{array}{l}24 \pm 16^{a} \\
12 \pm 3^{a}(50,00)\end{array}$ \\
\hline
\end{tabular}


Desinfectantes en aerosol como alternativa de control ambiental - A. Costa et al

respectivamente. Los principios activos de los productos utilizados están relacionados a alcoholes, amonio cuaternarios y fenoles, que son conocidos antifúngicos, lo que podría explicar el efecto inhibidor (Dvorak, 2008). Sin embargo, son compuestos, por definición, que tienen toxicidad no selectiva. Por ello, su recomendación para ser aplicados en superficies y ambientes, aunque algunos pueden ser utilizados como antisépticos (Frioni, 2011).

La temperatura y humedad en las habitaciones de las viviendas se muestran en la Tabla 4. Los valores de temperatura estuvieron dentro de un rango de 11 a $19,5^{\circ} \mathrm{C}$ y el porcentaje de humedad relativa varió entre un 64 a 85\%. Estos parámetros son adecuados para el desarrollo de hongos ambientales (Bueno et al., 2003, Afsal et al., 2004).

En relación a las encuestas realizadas, los datos están detallados en la Tabla 5 . El $89 \%$ de los encuestados $(n=46)$ conocía la existencia de desinfectantes ambientales en aerosol. El 78\% de la población estudiada $(n=41)$ conocía el Lysoform ${ }^{\circledR}$, seguido por Glade $\AA$ antitabaco y Ayudin ${ }^{\circledR}$. El producto Procenex ${ }^{\circledR}$ fue el menos conocido. Dentro de los productos más utilizados, el $54 \%$ de los encuestados $(n=35)$ indicó el producto Lysoform ${ }^{\circledR}$. La mayoría de los consumidores eligieron a los desinfectantes por lo que conocen y los medios de comunicación influyeron en el 29\% ( $n=35)$ de los consumidores al momento de elegir el desinfectante. El amplio conocimiento del Lysoform ${ }^{\circledR}$ en aerosol pudo deberse a las diversas propagandas del producto. El mismo se vende en Argentina desde 1970. Sin embargo, su relanzamiento en 1998, como un desinfectante de ambientes y superficies para uso diario en el hogar, generó conciencia sobre la existencia de gérmenes en el hogar y la importancia de mantener este último no sólo limpio, sino también desinfectado (Anónimo, SA). Por su parte, el nulo conocimiento del Procenex® pudo responder a que no se vendía ese producto en muchos supermercados de la ciudad.

Aunque le estudio se realizó con un muestreo gravimétrico, la carga de hongos ambientales/placa promedio fue disminuida con los diferentes productos desinfectantes en aerosol. Por ello, es recomendable la utilización, en una habitación, de los productos evaluados a fin de disminuir la carga de hongos ambientales y así reducir el efecto perjudicial de estos hongos sobre las personas que utilizan la habitación. Sin embargo, dada la cierta toxicidad de algunos principios activos que tienen estos productos desinfectantes en aerosol sobre las personas, es preferible su utilización cuando no haya personas en la habitación.

Tabla 4. Temperatura y humedad presente en las distintas habitaciones al momento del muestreo.

\begin{tabular}{|l|c|c|c|c|c|c|}
\hline \multirow{2}{*}{$\mathbf{N}^{0}$ Muestreo } & \multicolumn{6}{|c|}{ Temperatura ( $\left.{ }^{\circ} \mathrm{C}\right) \begin{array}{c}\text { y humedad relativa \% (RH) de las habitaciones de } \\
\text { las casas muestreadas }\end{array}$} \\
\cline { 2 - 7 } & \multicolumn{2}{|c|}{ Casa 1 } & \multicolumn{2}{c|}{ Casa 2 } & \multicolumn{2}{c|}{ Casa 3 } \\
\cline { 2 - 7 } & ${ }^{\circ} \mathrm{C}$ & $\mathrm{RH}$ & ${ }^{\circ} \mathrm{C}$ & $\mathrm{RH}$ & ${ }^{\circ} \mathrm{C}$ & $\mathrm{RH}$ \\
\hline 1 & 19,5 & 85 & 17,4 & 79 & 15,9 & 69 \\
2 & 11 & 66 & 14,8 & 65 & 11,8 & 80 \\
3 & 16 & 70 & 18,1 & 82 & 12,4 & 67 \\
4 & 16 & 66 & 16 & 71 & 13,6 & 64 \\
\hline Media & 15,6 & 71,75 & 16,6 & 74,25 & 13,4 & 70 \\
\hline
\end{tabular}


Tabla 5. Datos obtenidos de las 46 personas encuestadas sobre el uso de los desinfectantes ambientales.

\begin{tabular}{|c|c|}
\hline Pregunta & Respuestas (número de personas) \\
\hline \multirow[t]{2}{*}{ ¿Conoce algún desinfectante ambiental? } & Si (41) \\
\hline & No: 5 \\
\hline \multirow[t]{4}{*}{ ¿Cuál? } & Glade $\circledast$ antitabaco: 7 \\
\hline & Lysoform®: 32 \\
\hline & Ayudin ${ }^{\circ}: 2$ \\
\hline & Procenex®: 0 \\
\hline \multirow[t]{4}{*}{ ¿Cuál utiliza? } & Glade $®$ antitabaco : 9 \\
\hline & Lysoform®: 19 \\
\hline & Ayudin $®: ~ 6$ \\
\hline & Procenex®: 1 \\
\hline \multirow[t]{4}{*}{ ¿Por qué lo elige? } & Más económico: 6 \\
\hline & Se lo recomendaron: 4 \\
\hline & Es el que conoce: 15 \\
\hline & $\begin{array}{l}\text { Por los buenos resultados que se explicitan en los } \\
\text { medios de comunicación: } 10\end{array}$ \\
\hline
\end{tabular}

\section{AGRADECIMIENTOS}

Los autores agradecen al Dr. Leandro Marco y al Programa «Salud Para Todos» por el apoyo y el espacio físico para el desarrollo del trabajo en el marco del PID- UNER «Epidemiología del asma bronquial en la población infanto-juvenil de Concepción del Uruguay». 
Desinfectantes en aerosol como alternativa de control ambiental - A. Costa et al

\section{REFERENCIAS}

Afzal, M.; Mehdi, F.S.; and Siddiqui, Z.S. (2004). Effectof relative humidity and temperature on airborne fungal allergens of Karachi city. Pak. J. Biol. Sci. 7: 159-162.

Albright, D.M. (2001). Human health effects of airborne mycotoxins exposure in fungi contaminated indoor environment. Prof. Safety: 26-28.

Anónimo. (SA). Lysoform. Acceso: Noviembre, 2014. http://www.topbrandsargentina.com.ar/ pdf\%20Volumen2/lysoform.pdf

Auditoría ciudadana. (2006). Informe sobre la Calidad de las Prácticas Democráticas de Concepción del Uruguay Provincia de Entre Ríos República Argentina. Acceso: Noviembre, 2014. http://www. auditoriaciudadana.com.ar/sitio/informes/ concepcion_del_uruguay.pdf

Barnes, C.; Dinakar, C.; Reddy, M.; and Portnoy, J. (2006). Frequency of fungi in homes of pediatric allergy patients. J. World Allergy Org. 18/5: 197-202.

Bueno, D.J., Silva, J.O. y Oliver, G. (2003). Hongos ambientales en una biblioteca: un año de estudio. Anal. Doc. 6: 27-34.

Burge, H.A.; Piernon, D.L.; Groves, T.0.; Strawn, K.F.; and Mishra, S.K. (2000). Dynamic of airborne population in a large office building. Current Microbiol. 40: 10-16

Buttner, M.P.; Willeke, K.; and Grinshpun, S.A. (1996). Sampling and analysis of airborne microorganisms. In: C.J. Hurst (Ed.). Manual of Environmental Microbiology. American Society of Microbiology, Washington, D.C. pp. 629-640.

Duffau T., G. (1999). Tamaño muestral en estudios biomédicos. Rev. chil. Pediatr. 70: 314-324.

Dvorak, G. (2008). Disinfection 101. The Center for Food Security and Public Health. lowa State University. Acceso: Noviembre, 2014. http://www.cfsph.iastate.edu/ Disinfection/Assets/Disinfection101.pdf

Ernst, R.A. (1987). Microbiological monitoring of hatchery and hatching egg sanitation. World's Poult.
Sci. J. 43: 56-63.

Frioni, L. (2011). Microbiología: básica, ambiental y agrícola. Orientación Gráfica Editora, Buenos Aires.

Horner, W.E.; Helbling, A.; Salvaggio, J.E.; Lehrer S.B. (1995). Fungal allergens. Clin. Microbiol. Rev. 8: 161-170.

INDEC. (2008). Estimaciones de población total por departamento y año calendario Período 20012010.Serie № 34 Análisis demográfico. Acceso: Noviembre, 2014. http://www.indec.mecon.ar/ nuevaweb/cuadros/2/estimaciones-serie34.pdf

Lozano, A.; Sasia, L.; Alvarez, J.S.; Saranza, R.J.; Croce, J.S.; y Croce, V.H. (2003). Prevalencia de la sensibilización a los hongos anemófilos en niños con rinitis alérgica. Alerg. Inmunol. Clin. 20(4): 124-127.

Madigan, M.T.; Martinko, J.M.; y Parker, J. (2004). Biología de los microorganismos. Prentice Hall, Madrid, España.

Portnoy, J.M.; Kwak K.; Dowling, P.; Vanosdol, T.; and Barnes, C. (2005). Health effects in indoor fungi. Ann. Allergy Asthma Immunol. 94: 313-320.

Rossman, A.J., and Chance, B.L. (1998). Workshop Statistics: discovery with data and Minitab. SpringerVerlag New York Inc.

Senent Sánchez, C.J. (2012). Alergia a la humedad ¿qué son los hongos y cómo evitarlos? En J.M. Zubeldía, M.L. Baeza, I. Jáuregui, y C.J. Senent (eds). Libro de las enfermedades alérgicas de la Fundación BBVA. Fundación BBVA, Bilbao. pp.97-105

Sneller, M.R.; and Roby, R.R. (1979). Incidente of fungal spores at the homes of allergic patients in an agricultural community.l. A 12-month study in and out of doors. Ann. Allergy 43: 225-228.

Strass, M.D.; Ardusso, L.R.F.; y Crisci, C.D. (2002) Prevalencia de sensibilidad a aeroalergenos en pacientes con rhinitis $\mathrm{y} / 0$ asma en el sur de Misiones y noreste de Corrientes, Argentina. Arch. Alergia Inm. Clín. 33: 47-52. 
Tormo-Molina, R.; Gonzalo-Garijo, M.A; and Yang, C.S.; and Johanning, E. (1997). Airborne fungi Fernández-Rodríguez, S.; Silva-Palacios, I. and mycotoxins. In C.J. Hurst, G.R. Knudsen, (2012). Monitoring the occurrence of indoor fungi Mclnerney, L.D. Stetzenbach, W.V. Walter (ed). Manual in a hospital. Rev. Iber. Micol. 29: 227-234. of Environmental Microbiology. ASM Press. USA. pp. 651-660. 